\title{
Implementación del sistema ABCD en el CRAI de la Universidad de Cienfuegos
}

\author{
Ennia De la Cruz Medina \\ Daymé García Valdés \\ Elvys Chapis Cabrera
}

Centro de Recursos para el Aprendizaje y la Investigación, Universidad de Cienfuegos - UCF, Cuba

COMMUNICATION

\begin{abstract}
Resumen
Informa diversos aspectos sobre la experiencia de implementación del sistema ABCD en el Centro de Recursos para el Aprendizaje y la Investigación de la Universidad de Cienfuegos.
\end{abstract}

\section{Palabras clave}

Sistemas de gestión bibliotecaria ; Automatización de bibliotecas ; Centro de Recursos para el Aprendizaje y la Investigación ; CRAI ; $A B C D$; Universidad de Cienfuegos ; Cuba

\section{Implementing ABCD system in the services of the CRAl of the Cienfuegos university}

\author{
Abstract \\ Informs severals aspects about implementation experience of ABCD system in the Information Resource Center for Learning \\ of the University of Cienfuegos.

\section{Keywords} \\ Library management systems; Library automation; Resource Center for Learning and Research; CRAl; ABCD; University of \\ Cienfuegos; Cuba
}

\section{Introducción}

Automatización de Bibliotecas y Centros de Documentación ( $A B C D$ ) es un aplicativo que aspira a proveer una herramienta integrada de gestión de bibliotecas que cubra todas los procesos principales, como: adquisiciones, desarrollo de bases de datos bibliográficas, administración de usuarios, registro de préstamos, control de publicaciones periódicas, y catálogo público.

Es en ese sentido que los especialistas del Centro de Recursos para el Aprendizaje y la Investigación de la Universidad de Cienfuegos "Carlos Rafael Rodríguez", aprobaron la implementación de dicha herramienta con el propósito de digitalizar los servicios bibliotecarios del mismo.

Se comentan a continuación las bondades y aspectos destacables de la herramienta, que motivaron su elección y adopción por nuestro centro.

\section{Aspectos generales}

ABCD constituye una solución tecnológica tanto para los Centros de Recursos de Aprendizaje y la Investigación (CRAI) como para los Centros de Documentación especializados. El aplicativo está constituido por un conjunto de módulos relativamente independientes, que pueden cooperar e interactuar entre ellos. En ese sentido es un software avanzado y apto para ambientes exigentes que requieren de aplicaciones escalables acorde al 
incremento progresivo de sus demandas. En ese sentido es posible automatizar completamente una biblioteca pequeña con todas las funciones necesarias, usando sólo el módulo central de tal forma que todas las funciones estén cubiertas con un mínimo de complejidad tecnológica.

La función de búsqueda se ofrece en un contexto mucho más amplio de acceso a la información, como parte de la página del portal para los usuarios, facilitando el acceso a otros recursos de información (ejemplo Google, Medline, etc.), y comunicación (anuncios y alertas internas) proyentándose a funciones de tipo Web 2.0. Cuenta para ello con un administrador que permite diseñar la estructura y los componentes de la página principal de ABCD.

El sistema de control de publicaciones periódicas ofrece una herramienta avanzada de gestión, independientemente de su versión (impresas o electrónicas) o periodicidad, facilitando una opción de catálogo para estas.

El módulo de préstamos ofrece un manejo avanzado con algunas características extra para organizaciones más grandes y complejas. Provee además un módulo dirigido a los usuarios, basada en tecnología de servicios Web, que puede ser usado para reemplazar los módulos integrados de préstamo en caso de contar con muchas dependencias y altos volúmenes de transacciones.

El sistema tiene partes relativamente independientes, como es el caso de la integración de aplicaciones ofimáticas paquetes de automatización de oficinas (Open Office, Microsoft Office, etc.) con vínculos obvios para operar en conjunto

El módulo de estadísticas, al igual que el módulo circulación y préstamos, puede operar sobre cualquier base de datos, y además ofrecefrecuperación avanzada basada en la Web. El módulo de control de publicaciones periódicas administra bases de datos CDS/ISIS dentro o fuera del contexto de ABCD.

\section{Una herramienta orientada para el especialista}

$A B C D$ ofrece una herramienta para los especialistas en gestión bibliotecaria, antes que para los expertos en tecnologías de la información y comunicación. Esto es posible ya que ABCD adoptando como punto de partida los principios y criterios de la bibliotecología y las ciencias de la información.

En ese sentido cada entidad puede ser formada mediante el uso del lenguaje de formateo CDS/ISIS. Cualquier especialista en gestión bibliotecaria está perfectamente capacitado para comprender y utilizar todos los campos de la base de datos $A B C D$, alcanzando resultados avanzados de sistematización.

Otro aspecto importante es que ABCD es una herramienta idónea para países en desarrollo, dado:

- El bajo nivel de habilidades en Tecnologías de Información y Comunicación (Tics), los especialistas pueden resolver sus problemas evitando arquitecturas de software innecesarias, mientras disponen de flexibilidad dentro del software.

- La baja demanda de ancho de banda y conectividad: mediante el uso de técnicas web como AJAX y JavaScript, permite que el tráfico de datos entre el cliente y el servidor se mantenga al mínimo, lo que permite al equipo local (en el 'lado del cliente') procesar una mayor cantidad de datos sin recurrir constantemente al servidor Gestão da informação ; Gestão do conhecimento ; Produtos informacionales ; Serviços informacionales ; Estudos de caso ; Centro de Informação e Gestão Tecnológica ; Guantánamo ; Cuba

ABCD es heredero de la familia CDS/ISIS y cuenta con el respaldo tecnológico de la Biblioteca Virtual en Salud - BIREME.

\section{Requisitos de implementación}

$A B C D$ requiere de algunas condiciones específicas de hardware y software: 


\section{Hardware}

- Procesador a partir de un Core 2 Duo.

- Memoria a partir de 512 RAM.

- Disco de 80 GB o más.

\section{Software}

- Linux basado en Debian de 64 bit.

- Apache.

- Mysql.

\section{Conclusiones}

El diseño e implementación de ABCD en el CRAI de la Universidad de Cienfuegos constituye un hito en el desarrollo del centro y un hecho trascendente en la actividad de sus especialistas en gestión bibliotecaria.

La herramienta contribuye además con la propagación selectiva de la información entre y nuestros usuarios y los tros centros de nivel superior del país.

Finalmente, ABCD promueve el acceso a las bases de datos remotas sin requerir la presencia física del usuario en el CRAI.

\section{Bibliografía}

Calero Yera, Esmeralda, Guevara Sánchez, Danielkys, \& Caballero Zamora,Deysis. (2011). Jornada Nacional Virtual de Ciencias de la Información en Salud. Centro Provincial de Información de Ciencias Médicas. Recuperado a partir de http://escay@finlay.cmw.sld.cu

Fernández, Gladys, \& Lenzo, Nancy. (2010, julio). Software ABCD (Automatización de Bibliotecas y Centros de Documentación): nuevos desafíos para las bibliotecas argentinas. Biblioteca del Centro Médico de Mar del Plata. Recuperado a partir de http://eprints.rclis.org/14815

Macías González, Julio. (2011). Servicios Bibliotecarios a través de Internet. Recuperado a partir de http://www.sedic.es/autoformacion/servicios bibliotecarios/index.htm

Merlo Vega, José Antonio. (2000). Servicio Bibliotecario de Referencia. Universidad de Murcia. Recuperado a partir de http://www.um.es/fccd/anales/ad03/AD07-2000.PDF

OSS4lib. (s. f.). Portal de Sistemas de Gestión de Bibliotecas Open Source. Recuperado a partir de http://www.oss4lib.org/ 


\section{Datos de las autoras}

\section{Ennia De la Cruz Medina}

Licenciada en Gestión de la Información en Salud (2009). Ha ofrecido cursos, Talleres y conferencias sobre Alfabetización Informacional, Gestores de Referencias Bibliográficas, Metabuscadores, Bases de Datos, Se desempeña como Especialista en procesamiento y análisis de la información en el Centro de Recursos para el Aprendizaje y la Investigación (CRAl) de la Universidad de Cienfuegos "Carlos Rafael Rodríguez". ecruz@ucf.edu.cu

\section{Daymé García Valdés}

Licenciada en Comunicación Social de la Universidad de Cienfuegos (2012). Ha ofrecido cursos, Talleres y conferencias sobre Alfabetización Informacional, Gestores de Referencias Bibliográficas, Metabuscadores, Bases de Datos, Revistas Científicas y Sitios Científicos. Se desempeña como Especialista en procesamiento y análisis de la información en el Centro de Recursos para el Aprendizaje y la Investigación (CRAI) de la Universidad de Cienfuegos "Carlos Rafael Rodríguez".

dgvaldes@ucf.edu.cu

\section{Elvys Chapis Cabrera}

Licenciada en Información Científico Técnica y Bibliotecología de la Universidad de la Habana en el año 1994. Con 20 años de experiencia laboral. Ha ofrecido cursos, Talleres y conferencias sobre, Alfabetización Informacional, Gestores de Referencias Bibliográficas, Metabuscadores, Bases de Datos, Revistas Científicas, Sitios Científicos y Sistemas de Gestión de Información. Se categorizó como profesor en el año 2006, vinculándose a la docencia desde entonces. Se desempeña como Especialista en análisis, servicio y procesamiento de la información en el Centro de Recursos para el Aprendizaje y la Investigación (CRAI) de la Universidad de Cienfuegos "Carlos Rafael Rodríguez".

echapis@ucf.edu.cu

Recibido - Received : 2014-10-01

Aceptado - Accepted: 2015-03-30

\section{$(\mathrm{cc})$ BY}

This work is licensed under a Creative Commons Attribution 4.0

United States License.

\section{UILIS D-Solf}

This journal is published by the University Library System of the University of Pittsburgh as part of its D-Scribe Digital Publishing Program and is cosponsored by the University of Pittsburgh Press. 